\title{
Microscopic foundations of the Rayleigh law of hysteresis
}

\author{
Stefano Zapperi ${ }^{\mathrm{a}, *}$ Alessandro Magni ${ }^{\mathrm{b}}$ Gianfranco Durin ${ }^{\mathrm{b}}$ \\ a INFM sezione di Roma 1, Dipartimento di Fisica, Università "La Sapienza", P.le \\ A. Moro 2, 00185 Roma, Italy \\ ${ }^{\mathrm{b}}$ Istituto Elettrotecnico Nazionale Galileo Ferraris and INFM, Corso M. d'Azeglio \\ 42, I-10125 Torino, Italy
}

\begin{abstract}
The hysteresis properties of ferromagnetic materials at low field are described by the Rayleigh law. We analyze the problem in the light of modern statistical mechanics models of hysteresis. In particular, we compute the demagnetization curve and derive the Rayleigh parameters $a$ and $b$ in the random-field Ising model and in a model of domain wall depinning. In the random-field Ising model the Rayleigh law is obeyed only in the disorder dominated phase, while in the low disorder phase it is not possible to demagnetize the sample. This approach allows us to link $a$ and $b$ to microstructural parameters, such as the domain wall energy, the internal disorder or the exchange interactions. Finally, our results are compared with experiments.
\end{abstract}

Key words: Hysteresis, Domain wall dynamics

PACS: 75.60.Ej, 64.60.Ht, 68.35.Ct, 75.60.Ch

\section{Introduction}

In 1887 Lord Rayleigh analyzed the hysteresis properties of ferromagnetic materials at low fields, close to the demagnetized state [1-3]. When the field is cycled between $-H_{m}$ and $H_{m}$, the magnetization $M$ is found to follow a simple quadratic law $M=\left(a+b H_{m}\right) \pm b\left(H_{m}^{2}-H^{2}\right) / 2$, where the signs \pm distinguish the upper and lower branch of the loop. The area of the loop can easily be computed and is given by $W=(4 / 3) b H_{m}^{3}$. In addition, the response of the system to a small field change, starting from the demagnetized state is given

\footnotetext{
* Corresponding author. Phone: +390649913437 Fax:+39064463158

Email address: zapperi@pil.phys. uniroma1.it (Stefano Zapperi).
} 
by $M_{m}=a H_{m} \pm b H_{m}^{2}$. The Rayleigh law has been shown to hold in several ferromagnetic materials [4] and it has been also widely applied to describe ferroelectric materials $[5,6]$. A few papers have reported significant deviations from the simple quadratic law but no explanation has been provided [7].

In 1942 the Rayleigh law was interpreted by Néel in terms of the motion of a point (i.e. a rigid domain wall) in a random energy landscape, whose statistical properties determine the value of $a$ and $b$ [8]. In particular, $a$ is associated to reversible motions inside one of the many minima of the random potential, while $b$ describes irreversible jumps between different valleys. Several generalizations of this approach have been proposed, considering more refined forms for the random energy landscape [9-13]. These approaches assume a single rigid domain wall and thus do not consider the effect of domain wall bowing and the interactions between different domains. When this effects are important it is hard to link the Rayleigh law to the microstructural properties of the material, such as dimensionality, chemical composition or interaction types.

Here, we reconsider all these problems in light of novel statistical mechanics approaches to hysteresis. In particular we compute the Rayleigh parameters $a$ and $b$ in the random-field Ising model [14] and in a model of domain wall depinning [15-21]. This approach allows us to link $a$ and $b$ to microstructural parameters, such as the domain wall energy, the internal disorder or the exchange interactions.

\section{Models}

A microscopic theory of hysteresis should in principle recover the Rayleigh law from the collective properties of interacting magnetic moments. The Rayleigh parameters would then be expressed in terms of micromagnetic parameters, that are available in the literature for a variety of ferromagnetic materials. Ferromagnets can in general be described by a locally varying magnetization $M_{i}(\vec{r})$ evolving under the action of the externally applied field. The evolution of $M_{i}$ is ruled by appropriate relaxation equations that can be written in terms of the micromagnetic energy $[2,3]$. Considering for simplicity a uniaxial material, we can write the energy as

$$
E=\sum_{i=1}^{3} \int d^{3} r\left[A\left(\vec{\nabla} M_{i}\right)^{2}+K\left(M_{i} n_{i}\right)^{2}-\mu_{0} M_{s}\left(H_{i}+H_{d e m}^{(i)}\right) M_{i}\right]
$$

where $A$ is the exchange interaction, $K$ is the anisotropy constant, $n_{i}$ is the anisotropy axis, and $H_{d e m}^{(i)}$ is the component $i$ of the demagnetizing field and 
$M_{s}$ is the saturation magnetization.

An essential contribution to the properties of the hysteresis loops is given by disorder, due to crystal imperfections, internal stresses, non magnetic impurities, that are present in most magnetic materials. Néel recognized this fact and replaced the micromagnetic free energy by a random function of the magnetization (i.e. $\left.E=E_{R}(M)-H M\right)$ [8]. The approximation is rather drastic but makes the problem analytically tractable. In general we can model the disorder by quenched local fluctuations of the micromagnetic parameters. Disordered interaction terms are conventionally denoted as random bonds $(A \rightarrow A(\vec{r}))$, random anisotropies $\left(n_{i} \rightarrow n_{i}(\vec{r})\right)$ and random fields $\left(H \rightarrow H_{\text {ext }}+h(\vec{r})\right)$. A complete solution of micromagnetic equations including disorder is a very complicated task. One should then resort to some kind of approximation. In recent years two main approaches have been undertaken to describe the magnetization properties of disordered materials.

The first approach takes into account the fact that in soft magnetic materials demagnetizing fields give rise to broad domains and the magnetization process is dominated by domain wall motion. One can thus reduce the problem to the motion of a flexible domain wall in a random potential. The domain wall contribution to the micromagnetic free energy can be expressed in term of the domain wall coordinates $z(\vec{x})$, inserting in Eq. (1) $M_{i}(\vec{r})=\delta_{i 3} M_{s} g\left(\left(r_{3}-\right.\right.$ $\left.z(\vec{x})) / \delta_{w}\right)$ in Eq. (1), where $\delta_{w} \simeq \sqrt{A / K}$ is the domain wall width and $g(x)=$ \pm 1 for $x \rightarrow \pm \infty[15,18,21]$ :

$$
E_{d w}=\int d^{2} x\left[\gamma_{w}(\nabla z(\vec{x}))^{2}-z(\vec{x}) \mu_{0} M_{s}\left(H+H_{d}(\{z(\vec{x})\})+V(\vec{x}, z(\vec{x}))\right]\right.
$$

where the domain wall energy is given by $\gamma_{w} \simeq \sqrt{A K}$, the stray field is given by $H_{d}=\mu_{0} M_{s}^{2} \int d^{2} x^{\prime} \partial z / \partial x^{\prime}\left(x-x^{\prime}\right) /\left|r-r^{\prime}\right|^{3}$ and $V$ is a random function taking into account all the disorder contributions. In the following we will consider $V$ as a superposition of pinning centers randomly distributed in space

$$
V(\vec{x}, z)=\sum_{p} f_{0} \exp -\left(\left(z-Z_{p}(\vec{x})\right) / \xi_{p}\right)^{2}
$$

where $f_{0}$ is the strength of each pinning center, of width $\xi_{p}$ located in $Z_{p}$. The effective form of the pinning potential is not essential as long as the interaction range $\xi_{p}$ is finite. This is not the case for pinning due to isolated dislocations, whose stress field decays as $1 / r$. One should notice, however, that dislocations are typically arranged in patterns where the stress field is screened, providing us with an effective correlation length. In the following, we will restrict our attention to cases in which the pinning correlation length is smaller than the domain wall width, so that we can replace $\xi_{p}$ by $\delta_{w}$. This case corresponds 
mainly to the effect of non-magnetic impurities (see Ref. [16] for a discussion of this point).

Models based on Eq. 2 have been used in the past to compute the coercive field [15,17], analyze thermal relaxation [16], explain the statistical properties of the Barkhausen noise [19-22] and describe magnetization creep in thin films [23].

An approach based on domain wall motion is not adequate to describe the magnetization properties of hard ferromagnets, where the presence of strong random anisotropies prevents the formation of extended domains. For this class of materials a description in terms of interacting spins seems more appropriate. Several disordered spin models have been proposed in the past to describe hysteresis. Among those, the simplest and most studied is the RFIM $[14,24]$, where a spin $s_{i}= \pm 1$ is assigned to each site $i$ of a $d$-dimensional lattice. The spins are coupled to their nearest-neighbors spins by a ferromagnetic interaction of strength $J$ and to the external field $H$. In addition, to each site of the lattice it is associated a random field $h_{i}$ taken from a Gaussian probability distribution with variance $R, P(h)=\exp \left(-h^{2} / 2 R^{2}\right) / \sqrt{2 \pi} R$. The Hamiltonian thus reads

$$
E=-\sum_{\langle i, j\rangle} J s_{i} s_{j}-\sum_{i}\left(H+h_{i}\right) s_{i}
$$

where the first sum is restricted to nearest-neighbors pairs. The dynamics proposed in Ref. [14] is such that the spins align with the local field

$$
s_{i}=\operatorname{sign}\left(J \sum_{j} s_{j}+h_{i}+H\right) .
$$

In this way a single spin flip can lead the neighboring spins to flip, eventually trigger an avalanche. Using this dynamics it has been shown that the RFIM displays a phase transition in $d \geq 3$ as a function of $R$ [14,24]. For $R<R_{c}$, the saturation loop has a discrete jump in the magnetization at $H= \pm H_{c}$. The jump disappears for $R>R_{c}$ and $R=R_{c}$ corresponds to a critical point and the model satisfies scaling laws $[14,24]$.

\section{Collective pinning effects in domain wall hysteresis}

The hysteresis properties of interfaces in random media have been studied in the past in the context of frictional sliding [25]. Similar studies for ferro-

magnetic domain walls has only been restricted to the case of high driving 
frequencies and large fields [26]. It is interesting to note, however, that interfaces in random media obey return point memory, a typical properties of ferromagnetic hysteresis. Here, we use a model based on Eq. (2) to analyze the Rayleigh law at low frequency. For the sake of simplicity we do not consider here demagnetizing fields, which are essential, however, to account for the large scale behavior of the magnetization [21]. When considering small scale displacements of the domain wall, we expect the domain wall energy (scaling as $q^{2}$ in Fourier space a deformation of wave vector $\vec{q}$.) to be more relevant of the stray field contribution (scaling as $q$ ). The following arguments can nevertheless be generalized including the effects of demagnetizing fields $[15]$.

The scaling properties of the Rayleigh parameters can be obtained using collective pinning theory $[15,16,23]$. The central concept is the identification of a coherence length $L_{c}$, defining a region of space where the domain wall moves freely from the pinning centers. The coherence length can be obtained comparing the domain wall energy with the disorder fluctuations over a region of length $L_{c}$, considering only small transverse wall deformations of the order of $\delta_{w}$

$$
\gamma_{w} \delta_{w}^{2} \sim f_{0}\left(n_{0} L_{c}^{2} \delta_{w}\right)^{1 / 2} \quad L_{c} \sim\left(\gamma_{w} \delta_{w}^{3 / 2}\right) /\left(n_{0}^{1 / 2} f_{0}\right)
$$

The depinning field $H_{c}$, which can be identified with the coercive field, is obtained comparing the pinning energy with the magnetostatic energy over a region of length $L_{c}[15,16,23]$

$$
\mu_{0} M_{s} H_{c} L_{c}^{2} \delta_{w} \sim f_{0}\left(n_{0} L_{c}^{2} \delta_{w}\right)^{1 / 2} \quad H_{c}=\left(n_{0} f_{0}^{2}\right) /\left(\gamma_{w} \delta_{w}^{2} \mu_{0} M_{s}\right)
$$

This expression for the coercive field recovers the result obtained in Ref. [15] when two dimensional domain vaulting is considered. In models based on a rigid domain wall $[8,10,11]$ the coercive field is instead proportional to the standard deviation of the pinning field (i.e. $H_{c} \propto f_{0} \sqrt{n_{0}}$ ).

Under the application of a small external field the "unpinned" regions of the domain wall will bow slightly and contribute to the magnetization [16]. The expression for the susceptibility $a=d M / d H$ is similar to the one found in textbooks when discussing reversible susceptibility $[2,3]$, with exception that the bowing length is now given by $L_{c}$,

$$
a \sim \mu_{0} M_{s}^{2} L_{c}^{2} / \gamma_{w} \sim\left(\gamma_{w} \delta_{w}^{3}\right) /\left(n_{0} f_{0}^{2}\right) .
$$

This result differs from estimates based on generalizations of Néel theory $[8,10,11]$ where $\gamma_{w}$ does not appear. 
The first correction to the linear susceptibility, and hence the parameter $b$, can be obtained noticing that a small applied field can in principle lead to local depinning events, thus increasing the coherence length [25]. Using the arguments reported in Ref. [25], one can show that, to lowest order in $H$, $L_{c}(H)=L_{c}(0)\left(1+c|H| / H_{c}\right)$ where $c$ is a numerical factor. Inserting this expression in Eq. (8) and expanding for small $H$, we obtain $b \sim a / H_{c}$. A similar expression was already reported by Néel in 1942 [8].

In order to test these consideration, we perform numerical simulations based on Eq. 2. The coordinates of the domain walls evolve according to an overdamped equation $\Gamma d z / d t=-\delta E / \delta z$, where $\Gamma$ is an effective viscosity. The equation of motion is discretized on a grid of size $50 x 50$ and solved by an adaptive-stepsize Runge-Kutta method. We use quasistatic driving condition, applying a field $H$ and integrating the motion until the domain wall comes to rest. The system is first demagnetized, with the successive application of positive and negative fields of decreasing amplitude, and then we cycle the field between $-H_{m}$ and $H_{m}$. The Rayleigh parameters are extracted from the scaling properties of the hysteresis loops (see Fig. 1) which are averaged over several realizations of the disorder. The procedure is repeated for different values of the domain wall energy $\gamma_{w}$ and in Fig 2 we show that simulations are in agreement with the scaling theory.

\section{Random-field Ising model}

The RFIM is probably the simplest model showing the combined effect of disorder and exchange interaction on the shape of the hysteresis loop. The model can be solved exactly in one dimension and minor loops can also be computed [27]. Recently we have been able to obtain exact results for the entire demagnetization process in $d=1$, including the Rayleigh laws. Here, we present numerical results for the Rayleigh laws in $d=2$ and $d=3$.

In $d=2$, we perform a perfect demagnetization and thus obtain unambiguously the demagnetized state for a given realization of the disorder. This is done in practice changing the field by precisely the amount necessary to flip the first unstable spin [24]. In this way, the field is cycled between $-H_{m}$ and $H_{m}$ and $H_{m}$ is then decreased at the next cycle by precisely the amount necessary to have one avalanche less than in the previous cycle. This corresponds to decrease $H_{m}$ at each cycle by an amount $\Delta H$, with $\Delta H \rightarrow 0^{+}$. We thus obtain the demagnetizing curve and extract the Rayleigh parameters close to the demagnetized state for a system of linear size $L=50$ (see Fig. 3) for different values of $R$. During demagnetization, in higher order loops we observe that the same set of spins flips at precisely the same field, despite the fact that the demagnetization proceeds and the initial state is different. 
Performing a perfect demagnetization, however, it is not possible to go to very large system sizes. We have thus resorted to a different algorithm which perform an approximate demagnetization: instead of cycling the field between $-H_{m}$ and $H_{m}$ we just flip the field between these two values. The no-passing rule [14] ensures that the system will go into the same states that would be obtained decreasing and increasing adiabatically $H$ between $-H_{m}$ and $H_{m}$. The only difference is that, after each cycle, we have to decrease $H_{m}$ by an arbitrary value $\Delta H$ (i.e. we can not perform the limit $\Delta H \rightarrow 0^{+}$exactly). We have confirmed by numerical simulations that the results obtained with an approximate demagnetization with $\Delta H<10^{-3}$ are in good agreement with the ones obtained under a perfect demagnetization.

An approximate demagnetization is then used in $d=3$, in order to analyze the effect of the phase transition on demagnetization and the Rayleigh law (the transition is not present in $d=1$, while in $d=2$ the issue is controversial). We find that demagnetization is possible only for $R>R_{c}$, where $R_{c}=2.16$ for $J=1$ [24]. For $R<R_{c}$ the demagnetization curve coincide with the saturation loop and it is thus not possible to define the Rayleigh parameters. We thus measure $a$ and $b$ for different values of $R>R_{c}$ and linear system sizes ranging from $L=25$ to $L=100$. The results show that $a$ and $b$ vanish for $R \rightarrow R_{c}$ and follows a scaling law $a \sim\left(R-R_{c}\right)^{\beta_{a}}$ and $b \sim\left(R-R_{c}\right)^{\beta_{b}}$, with $\beta_{a} \simeq \beta_{b} \simeq 0.5$. This result suggests that the demagnetization curve scales simply as $M=\left(R-R_{c}\right)^{1 / 2} m(H)$ for $H \rightarrow 0$. It is interesting to compare this result with the behavior expected for $H=H_{c}$ on the saturation loop: in that case the magnetization scales with an exponent $\beta \simeq 0.04$, while $\beta=1 / 2$ is valid only in mean-field theory.

\section{Experimental comparison and conclusions}

In order to test the result obtained above, we perform a set of experiments on magnetostrictive ribbons of $\mathrm{Fe}_{64} \mathrm{Co}_{21} \mathrm{~B}_{15}$ amorphous alloy under moderate tensile stress $[28,29]$. This material is characterized by extended domain walls and the statistical properties of its Barkhausen noise have been recently shown to be well described by models of domain wall pinning $[22,29]$. We thus expect that its hysteretic behavior could also be described by collective pinning theory.

The sample is first demagnetized and hysteresis loops are measured at low fields. We find that the Rayleigh law is not perfectly verified at very low fields, but the hysteresis cycle becomes parabolic at higher field. At very low fields the magnetization is completely reversible, then for higher fields a definite and reproducible magnetization jump appears. Finally, at still higher fields several jumps combined together give rise to what can be well approximated by a 
parabolic cycle. This behavior is summarized in Fig. 5a where we report the behavior of $M_{m} / H_{m}$ as a function of $H_{m}$. The linear behavior at high field is described the Rayleigh law and the parameters $a$ and $b$ are consistent with estimates obtained from the loop shape.

In order to understand this behavior we have simulated the domain wall model for a single realization of the disorder using a grid of linear size $L=100$. Also in this case, we observe at low fields a reversible response and a jump at higher fields. After the jump the hysteresis loop is well described by the Rayleigh law (see Fig. 5b). Notice that when we average the cycles over different disorder configurations, the jump disappears. Clearly, disorder averaging can not be performed directly for experimental data, unless different samples are used. One should thus keep this in mind when Rayleigh parameters are estimated.

In conclusions, we have discussed the occurrence of the Rayleigh law of hysteresis, analyzing two classes of models: disordered spin models and domain wall depinning models. This is a step towards the ambitious goal to recover hysteresis loops properties from the magnetic microstructure.

\section{References}

[1] L. Rayleigh, Philos. Mag., Suppl. 23 (1887) 225.

[2] G. Bertotti, Hysteresis in Magnetism, Academic Press, San Diego, 1998.

[3] S. Chikazumi, Physics of Magnetism, Wiley, New York, 1964.

[4] R. Vergne, J. C. Cotillard, J. L. Porteseil, Rev. Phys. Appl. 16 (1981) 449.

[5] D. Damjanovic, J. Appl. Phys. 82 (1997) 1788.

[6] D. Bolten, U. Böttiger, T. Schneller, M. Grossmann, O. Lohse, R. Waser, Appl. Phys. Lett. 77 (2000) 3830.

[7] G. Bertotti, F. Fiorillo, M. Pasquale, J. Appl. Phys. 69 (1991) 5930.

[8] L. Néel, Cah. Phys. 12 (1942) 1.

[9] K. H. Pfeffer, Phys. Stat. Sol. 21 (1967) 857.

[10] H. Kronmüller, Z. Angew. Phys. 30 (1970) 9.

[11] H. Kronmüller et al., J. Magn. Magn. Mat. 13 (1979), 53.

[12] H. Kronmüller, T. Reininger, J. Magn. Magn. Mat. 112 (1992) 1.

[13] A. Magni, C. Beatrice, G. Durin, G. Bertotti, J. Appl. Phys. 86 (1999) 3253.

[14] J. P. Sethna, K. Dahmen, S. Karta, J. A. Krumhansl, J. D. Shore, Phys. Rev. Lett. 70 (1993) 3347. 
[15] H. R. Hilzinger, H. Kronmüller, J. Magn. Magn. Mat. 2 (1976) 11.

[16] T. Nattermann, Y. Shapir, I. Vilfan, Phys. Rev. B 42 (1990) 8577.

[17] Y. Enomoto, J. Magn. Magn. Mat. 129 (1994) L146.

[18] S. T. Chui, Phys. Rev. B 51 (1995) 250.

[19] J. S. Urbach, R. C. Madison, J. T. Markert, Phys. Rev. Lett. 75 (1995) 4694.

[20] P. Cizeau, S. Zapperi, G. Durin, H. E. Stanley, Phys. Rev. Lett. 79 (1997) 4669.

[21] S. Zapperi, P. Cizeau, G. Durin, H. E. Stanley, Phys. Rev. B 58 (1998) 6353.

[22] G. Durin, S. Zapperi, Phys. Rev. Lett. 84 (2000) 4075.

[23] S. Lemerle, J. Ferré, C. Chappert, V. Mathet, T. Giamarchi, P. L. Doussal, Phys. Rev. Lett. 80 (1998) 849.

[24] O. Perkovic, K. A. Dahmen, J. P. Sethna, Phys. Rev. B 59 (1999) 6106.

[25] L. Bocquet, H. J. Jensen, J. Phys. I (France) 7 (1997) 1603.

[26] I. F. Lyuksyutov et al., Phys. Rev. B 59 (1999) 4260.

[27] P. Shukla, Phys. Rev. E 62 (2000) 4725.

[28] C. Appino, G. Durin, V. Basso, C. Beatrice, M. Pasquale, G. Bertotti, J. Appl. Phys. 85 (1999) 4412.

[29] G. Durin, S. Zapperi, J. Appl. Phys. 85 (1999) 5196. 

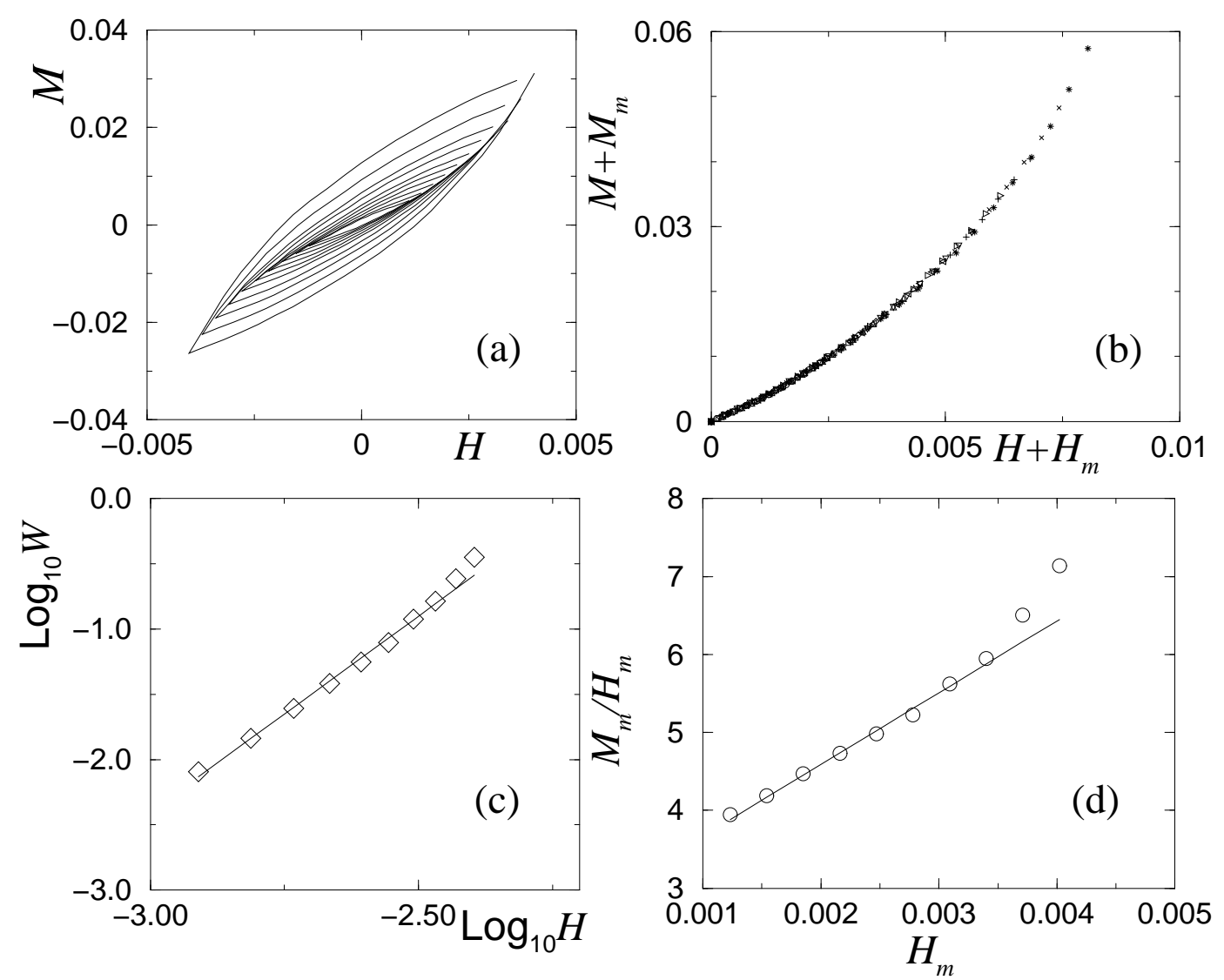

Fig. 1. The Rayleigh law computed in flexible domain wall in a random medium. (a) Hysteresis loop for different values of $H_{m}$. (b) The lower branch of the loops can be rescaled according to the Rayleigh law. (c) The area of the loop scales as $W \sim H_{m}^{3}$ (the line has a slope of 3. (d) The Rayleigh parameter can be also obtained by a linear fit of $M_{m} / H_{m}$ vs $H_{m}$. All units are arbitrary. 

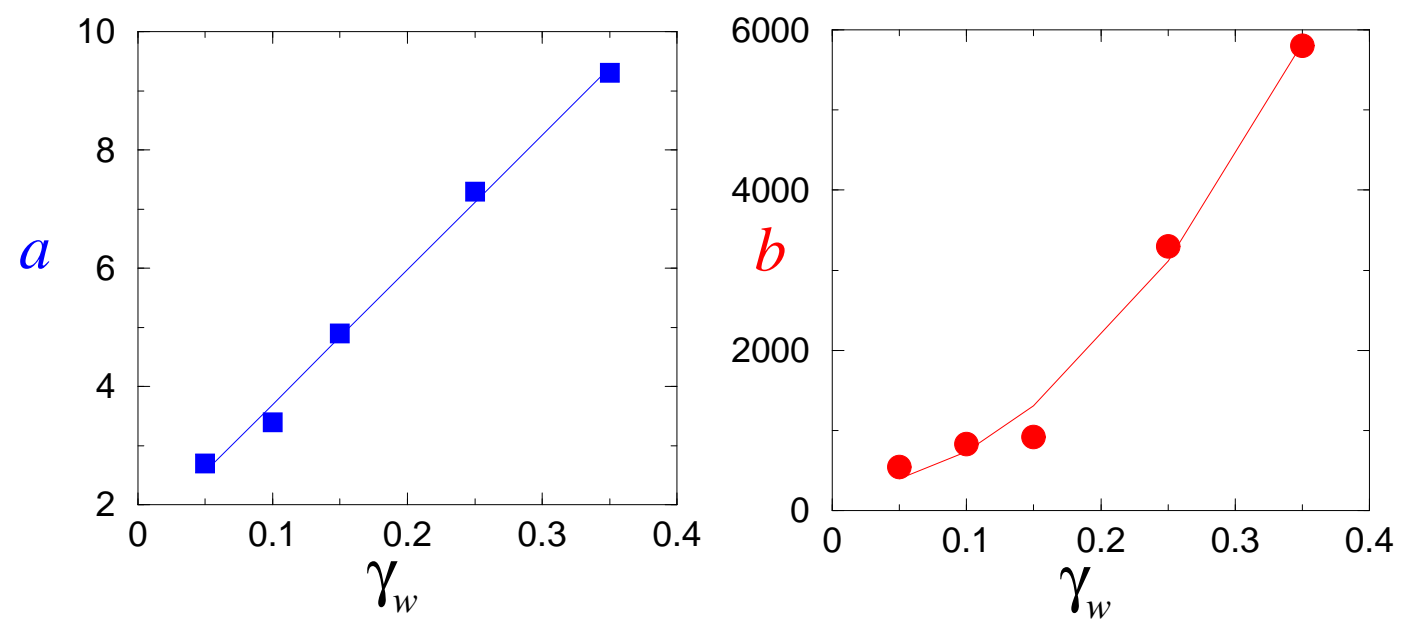

Fig. 2. The Rayleigh parameter $a$ and $b$ are computed for different values of the domain wall energy $\gamma_{w}$. The results satisfy the relation $a \propto \gamma_{w}$ and $b \propto \gamma_{w}^{2}$. All units are arbitrary. 


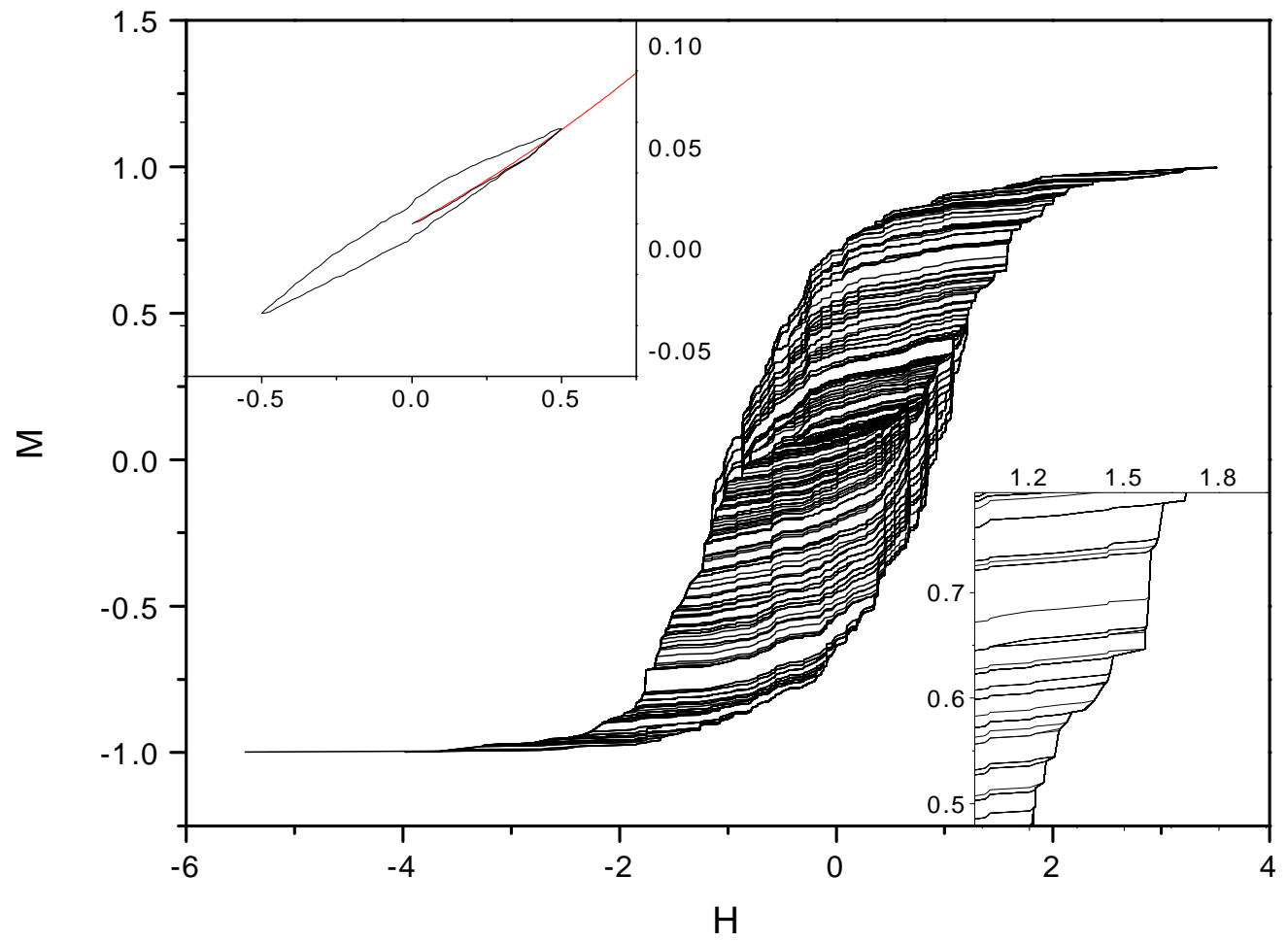

Fig. 3. An example of exact demagnetization process of the RFIM in $d=2$ for $R=1.64$. In the lower inset we show a detail of the main figure. In the upper inset we show the demagnetization curve and a small loop around the demagnetized state, both averaged over 30 realizations of the disorder. In the lower inset we show a detail of the main figure. All units are arbitrary. 


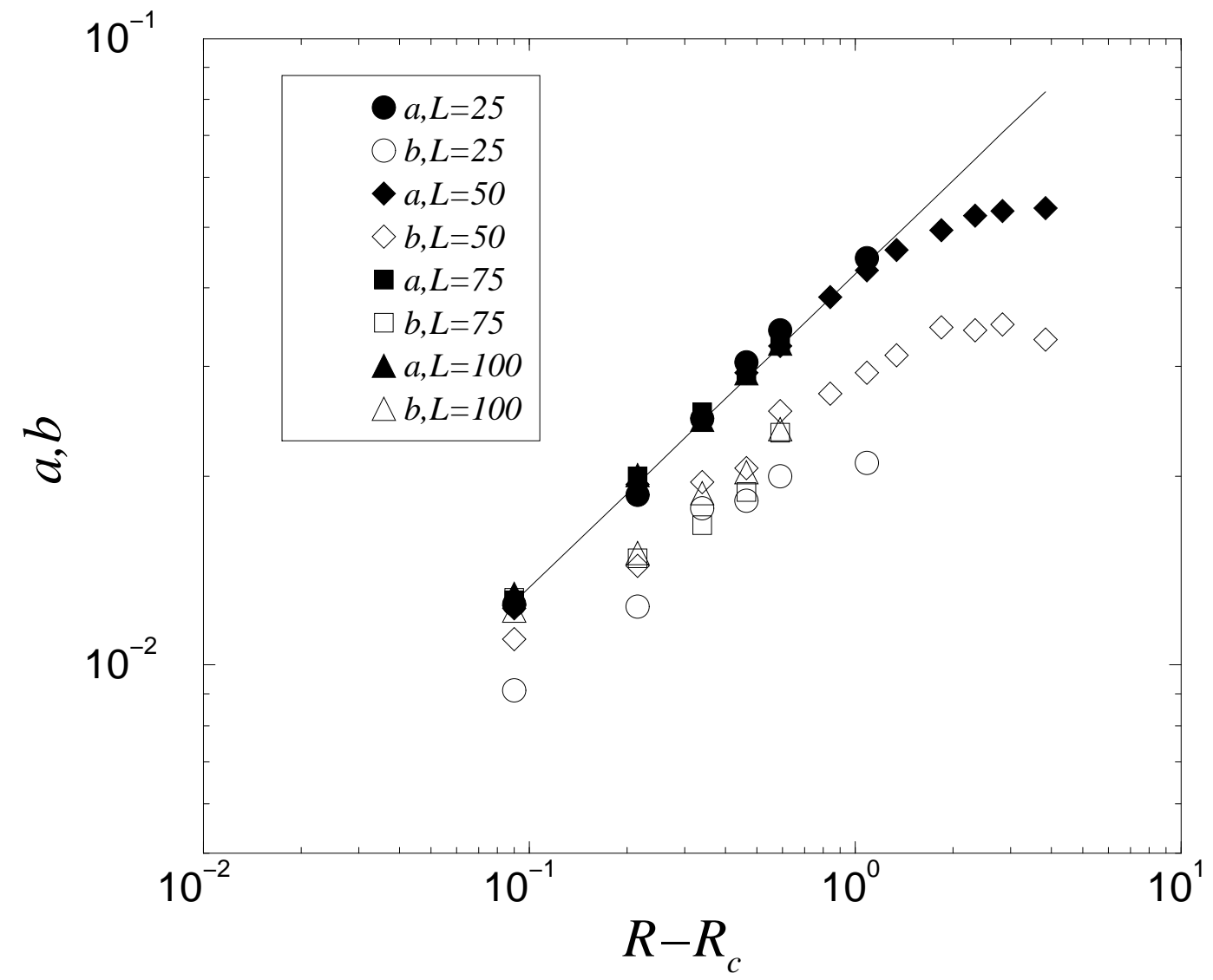

Fig. 4. The Rayleigh parameters $a$ and $b$ are computed for different values of the disorder $R$ in the RFIM in $d=3$, the results are the average over $N$ realization of the disorder: $N=100$ for $L=25 . N=50$ for $L=50, N=20$ for $L=75$ and $N=5$ for $L=100$. The Rayleigh law is only found for $R>R_{c}$ and the parameters scale to zero as power law when $R \rightarrow R_{c}$. All units are arbitrary. 

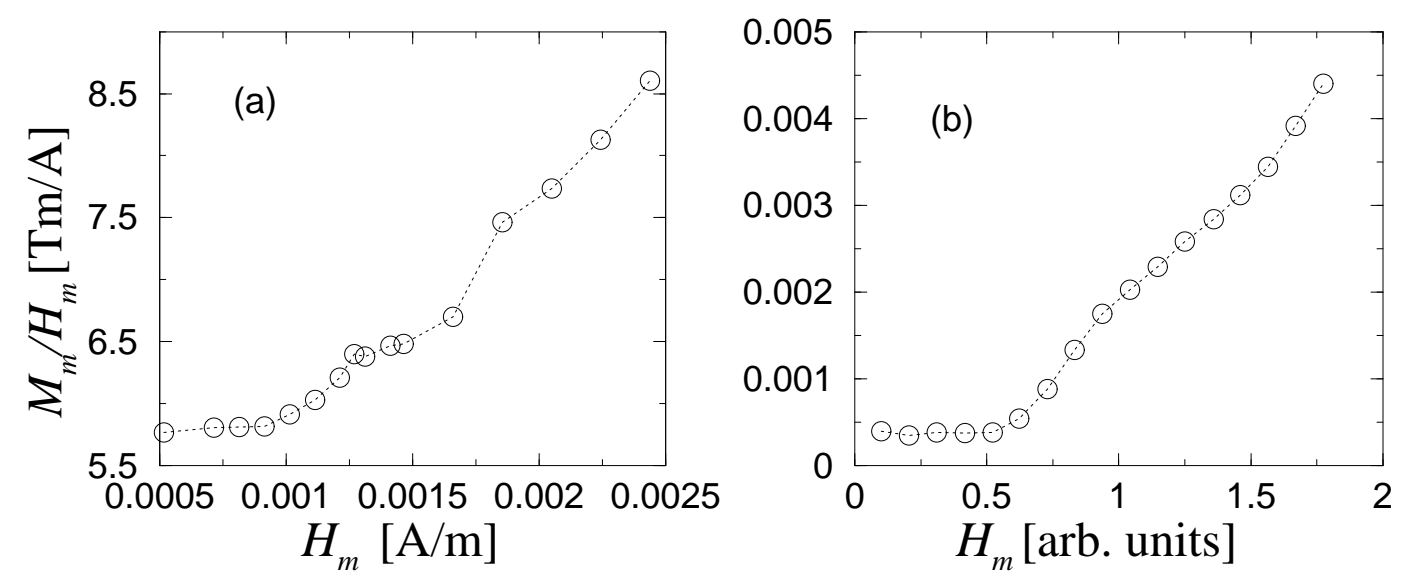

Fig. 5. The peak susceptibility $M_{m} / H_{m}$ as a function of $H_{m}$ : (a) for a single realization of the disorder in the domain wall model and (b) for a $\mathrm{Fe}_{64} \mathrm{Co}_{21} \mathrm{~B}_{15}$ amorphous ribbon. 